Kansas State University Libraries

New Prairie Press

\title{
ANALYZING SPLIT-PLOT ANDREPEATED-MEASURES DESIGNSUSING MIXED MODELS
}

\author{
Russ Wolfinger \\ Nancy Miles-McDermott \\ Jenny Kendall
}

Follow this and additional works at: https://newprairiepress.org/agstatconference

Part of the Agriculture Commons, and the Applied Statistics Commons

\section{(c) (1) $\Theta(9$}

This work is licensed under a Creative Commons Attribution-Noncommercial-No Derivative Works 4.0 License.

\section{Recommended Citation}

Wolfinger, Russ; Miles-McDermott, Nancy; and Kendall, Jenny (1992). "ANALYZING SPLIT-PLOT ANDREPEATED-MEASURES DESIGNSUSING MIXED MODELS," Conference on Applied Statistics in Agriculture. https://doi.org/10.4148/2475-7772.1405

This is brought to you for free and open access by the Conferences at New Prairie Press. It has been accepted for inclusion in Conference on Applied Statistics in Agriculture by an authorized administrator of New Prairie Press. For more information, please contact cads@k-state.edu. 


\title{
ANALYZING SPLIT-PLOT AND REPEATED-MEASURES DESIGNS USING MIXED MODELS
}

\author{
Russ Wolfinger, Nancy Miles-McDermott, and Jenny Kendall \\ SAS Institute Inc, SAS Campus Drive, Cary, NC 27513
}

\section{Abstract}

We first introduce the general linear mixed model and provide a justification for using REML to fit it. Then, for an irrigation example, we present several mixed models of increasing complexity. The initial model corresponds to a typical split-plot analysis. Next, we present covariance structures that directly describe the variability of repeated measures within whole plots. Finally, we combine the above types into more complicated mixed models, and compare them using likelihood-based criteria.

Some key words: Covariance structure, Mixed model, Restricted maximum likelihood

\section{Introduction}

The mixed model is a linear version of the signal-plus-noise model

$$
y=\mu+\epsilon
$$

where $\mu=X b$ and $\epsilon=Z u+e$. The signal is thus assumed to be a linear function of unknown fixed-effects parameters $b$, and the noise is decomposed into two additive terms. This decomposition permits you to model random effects in $u$ and additional error in $e$. Under the general assumption

$$
\operatorname{Var}\left[\begin{array}{l}
u \\
e
\end{array}\right]=\left[\begin{array}{cc}
G & 0 \\
0 & R
\end{array}\right]
$$

the mixed model provides a fair amount of flexibility in capturing the covariance structure of the noise. We recommend using restricted/residual maximum likelihood (REML) to estimate $G$ and $R$, and then computing estimates of $b$ and $u$ using these REML estimates. For a general introduction to this methodology, see McLean, Sanders, and Stroup (1991). 
In this article we discuss our preference of REML, and then demonstrate the mixed model's flexibility by applying it to data arising from two very common agricultural designs: split-plot and repeated-measures. Split-plot designs are handled effectively in $G$ and repeated-measures designs in $R$. It is even possible to combine the two designs into one complex mixed model. The $R$ matrix is also suited for modeling spatial variability. We use an example data set and the new SAS/STAT MIXED procedure to analyze these mixed models.

\section{Why REML?}

We recommend using REML instead of method-of-moments to fit mixed models. Method-of-moments procedures equate expected quadratic forms to observed quadratic forms and solve the resulting system of equations. ANOVA, MIVQUE, and MINQUE procedures are all examples of method-of-moments.

The method of moments is attractive because it makes no distributional assumptions about the data. This is in contrast to REML and maximum likelihood (ML), which by definition require the specification of a probability distribution for $u$ and $e$, usually normal. Also, method-of-moments procedures are often more simple computationally than the likelihood-based approaches.

However, the normal-theory REML and ML estimates have appealing asymptotic properties, even for nonnormal data (see Gourieroux, Monfort, and Trognon, 1984). REML can also be derived free of distributional assumptions by iterating the MIVQUE procedure. For discrete data, it is possible to fit generalized linear models with random effects and correlated errors (Wolfinger and O'Connell, 1992).

REML and ML estimates can be computationally intensive, although most problems can be performed with no more than an overnight turnaround on a PC. Large data problems, such as in genetics, remain computationally difficult, although progess is being made. Unbalanced data close the computational gap between REML and method-of-moments as evidenced by the complexity required in the article by Marta Remmenga at this conference. REML handles unbalance by constructing and optimizing the residual likelihood of the observed data-no observations are discarded as in some MANOVA procedures.

Several other arguments for preferring REML or ML to method-of-moments are provided by Harville (1977) and Searle et al. (1992). To summarize, REML has been 
shown in simulation studies to perform better in terms of bias and mean-squared error than method-of-moments (Swallow and Monahan, 1984). A Newton-type implementation of REML produces an asymptotic covariance matrix for the estimated parameters; this is absent in most method-of-moments procedures. Also, methodof-moments estimation becomes more difficult upon introduction of nontrivial structures for $G$ and $R$. Missing data complicates the construction of moment equations, whereas the likelihood of unbalanced data is usually easy to formulate. ML and REML have an asymptotic justification from a Bayesian viewpoint (Lindley, 1972, Section 11). Finally, REML and ML permit the use of likelihood-based criteria for model comparison (see Section 5 ).

REML is preferred to ML because it adjusts for bias in the estimation of $G$ and $R$. However, only ML is useful in deciding between different fixed-effects models.

\section{Example Data Set}

Line-source sprinkler irrigation data are presented in Hanks et al. (1980), and analyzed by Johnson et al. (1983), Stroup (1989), and in Example 16.6 of the PROC MIXED documentation, SAS Technical Report P-D29. Three cultivars (CULT) of winter wheat are randomly assigned to rectangular plots within each of three blocks (B). The nine plots are located side-by-side, and a line-source sprinkler is placed across the middle of them. Each plot is subdivided into twelve subplots, six to the north of the line-source, six to the south. The subplots closest to the line-source represent the maximum irrigation level (IRRIG=6), the two next-closest the nexthighest level (IRRIG=5), and so forth. The data are listed spatially in Table 1

\section{A Collection of Mixed Models}

Several types of mixed models are now applied to the example data set. Obviously some of the simpler models may not be appropriate because of the spatial nature of the data, but they are included for sake of illustration. For simplicity, the North vs. South effect is ignored throughout the analyses. 


\section{$4.1 \quad$ Split-Plot Models}

The traditional split-plot design with block B, whole-plot (ULT, and subplot IRRIG is fit in PROC MIXED as follows:

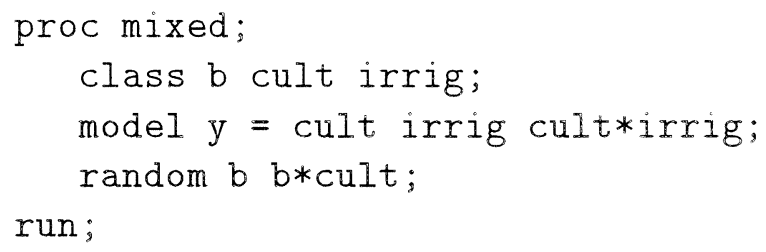

The syntax for PROC MIXED) is similar to that of PROC GLM, although the RANDOM and REPEATED statements are different. In PROC MIXED, the fixed-effects modeling in $X$ takes place in the MODEL statement, the random-effects modeling in $Z$ and $G$ takes place in the RANDOM statement, and variance modeling in $R$ takes place in the REPEATED statement. The $G$ matrix for this model is diagonal and contains the variance components for $\mathrm{B}$ and $\mathrm{B}^{*} \mathrm{CULT}$. The $R$ matrix is of the trivial form $\sigma^{2} I$-this is the default when you do not provide a REPEATED statement. The above code will compute REML estimates of the two variance components and $\sigma^{2}$.

The general mixed-model testing procedure described in the PROC MIXED) documentation replaces the typical construction of "Error A" and "Error B" to test whole-plots and subplots, respectively. In fact, the use of REML eliminates the need for a traditional ANOVA table, and PROC MIXED prints only the Type III $F$ statistics. Bear in mind that, except in special balanced cases, these $F$-statistics have only approximate $F$-distributions. The approximate $F$-tests are usually adequate, although further research is needed.

An equivalent way of specifying the the above model is the following:

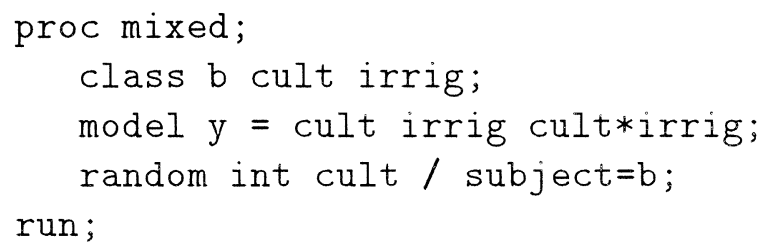

In this code, B has been "factored out" of the random effects and made into a special SUBJECT = effect. The SUBJECT = effect defines blocks of $G$, and this job may 
run faster than the previous one for larger problems. The estimates from both runs will be identical.

An interesting alternative to the traditional split-plot analysis is the following:

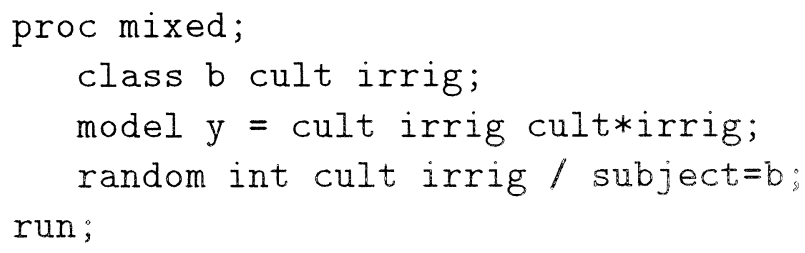

Note that $B^{*}$ IRRIG has been added as a random effect. Both George Milliken and Gary Richardson commented that this analysis would be appropriate for a stripblock design. Deborah Boykin and Brian Allen suggested that the inclusion of the extra term may be appropriate even in the usual split-plot situations.

\section{$4.2 \quad$ Repeated-Measures Models}

We now analyze the data as repeated measures within a row, with each row independent. Thus $R$ is assumed to be block diagonal, with each $12 \times 12$ block corresponding to a row. A typical analysis in this situation proceeds along the lines described in Jennrich and Schluchter (1986). That is, we select a covariance structure to model the variability of the data within a row. The following two examples fit structures corresponding to compound symmetry and unstructured:

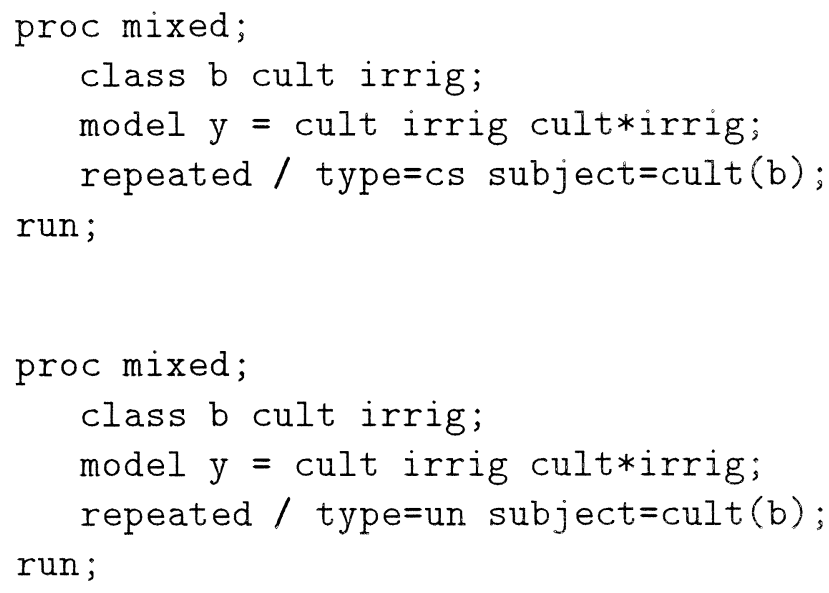


Note that SUBJECT=CULT(B) defines the rows as the blocks of $R$.

These two analyses correspond to the traditional univariate and multivariate approaches, respectively, to repeated measures. The mixed model method thus accomodates these two approaches as special cases. However, for this example, the $12 \times 12$ unstructured matrix has 78 unknown parameters, and is not practical. More parsimonious structures include factor-analytic, AR(1), and Toeplitz.

\subsection{Combination Mixed Models}

Split-plot and repeated-measures designs can be combined as follows:

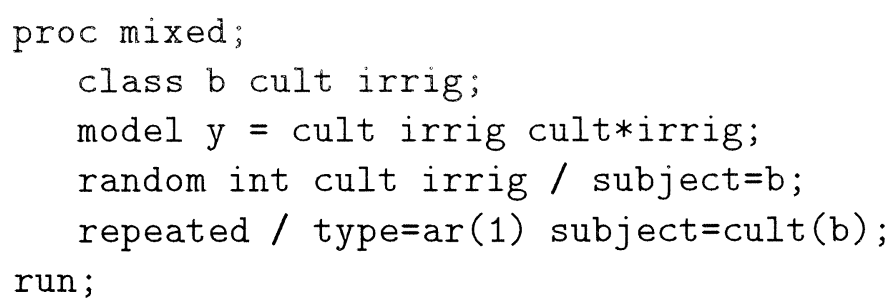

Other examples of combination mixed models are given by Stroup (1989) for these data and by Brian Allen at this conference for tree ring chronologies.

\subsection{Spatial Models}

For a description of spatial (kriging) models, see Marx and Thompson (1987) and Zimmerman and Harville (1991). The following is an example that models a spherical spatial correlation across the entire data set:

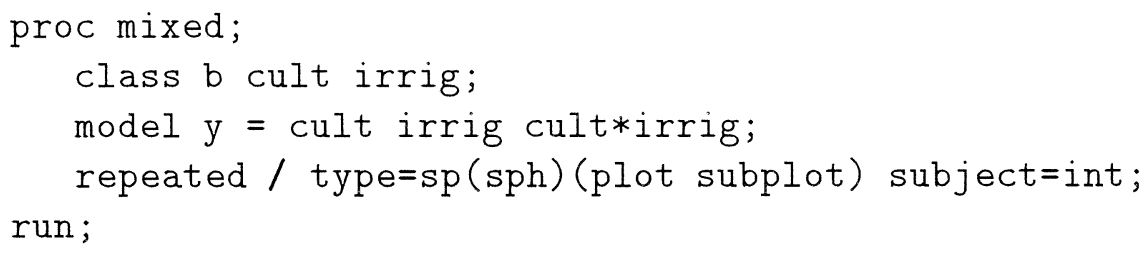


PLOT and SUBPLOT are used as coordinates to locate the individual data points. In some cases these coordinates should be rescaled to produce more sensible optimization problems. SUB.JECT $=$ INT is a device to specify the non-block-diagonal $108 \times 108 R$ matrix.

You can fit the quadratic surface model presented by Phil Cox at this conference by including the appropriate terms in the MODEL statement.

\section{Model Comparison}

We now fit several mixed models to the line-source sprinkler example data set and compare them using Akaike's information criterion (AIC, Akaike, 1974; Amemiya, 1980) and Schwarz's Bayesian criterion (SBC, Schwarz, 1978):

$$
\begin{aligned}
& A I C=\text { LogLikelihood }-q \\
& S B C=\text { LogLikelihood }-\frac{q}{2} \log n
\end{aligned}
$$

Here $q$ is the number of covariance parameters and $n$ is the number of observations.

Larger values of the criteria are preferred, and SBC favors more parsimonious models than does AIC. You can also perform likelhood ratio tests between models that are nested within each other by subtracting -2 times their respective log likelihoods.

Table 2 lists AIC, SBC, and -2 log likelihoods corresponding to REML and ML for a variety of mixed models fit to the example data. All of the mixed models have CULT, IRRIG, and CULT*IRRIG as fixed effects, and the various structures for $R$ and $G$ are listed in the first two columns. In the first column, which describes the $R$ matrix, I stands for the identity matrix times a residual variance component, CS for compound symmetry, AR(1) for first-order autoregressive, TOEP for Toeplitz, UN for unstructured, and SP for spatial. The symbol AR(1)*CULT indicates a heterogeneous $R$ matrix consisting of separate $\mathrm{AR}(1)$ parameters for each cultivar. This heterogeneity is specified in PROC MIXED by using the GROUP $=$ CULT option on the REPEATED statement. TOEP(4) is a Toeplitz matrix containing 0s in all but the first four principal bands. $\mathrm{UN}(1)$ in an unstructured matrix containing 0s everywhere except the main diagonal. All of the structures, including the spatial ones, are described in the PROC MIXED) documentation. The $G$ matrix for all of the models is either nonexistent or diagonal containing variance components. 
In actually comparing the models, REML AIC prefers the Toeplitz $R$ and B B*IRRIG $G$, while ML AIC prefers just the Toeplitz $R$ with no $G$. We encountered some difficulties with local maxima when using the Toeplitz structure, so these values may not be reliable. Both REML and ML SBC prefer the spatial power-law structure, which seems to be a reasonable model for these data.

Upon selection of a model, you can construct appropriate linear combinations and quadratic forms of the estimated fixed and random effects, e.g. least-squares means and Type III F-statistics.

\section{Summary}

Dr. Stu Hunter challenged us at this conference to improve our modeling of both signal and noise. With regards to signal, we should very often be searching for an appropriate differential equation. The linear fixed-effects term $X b$ of the mixed model frequently provides an adequate approximation to this relevant theoretical signal. With regards to noise, the random-effects-plus-error term $Z u+e$ of the mixed model is a useful departure from the iid-normal litany. Therefore, the general mixed model is certainly a valuable tool in meeting Dr. Hunter's challenge.

\section{References}

Akaike, H. (1974). A new look at the statistical model identification. IEEE Transaction on Automatic Control, AC-19, 716-723.

Amemiya, T. (1980). Selection of regressors. International Economic Review, 21, 331-354.

Gourieroux, C., Monfort, A., and Trognon, A. (1984) Pseudo maximum likelihood methods: theory. Econometrica 52, 681-700.

Hanks, R.J., Sisson, D.V., Hurst, R.L, and Hubbard K.G. (1980). Statistical analysis of results from irrigation experiments using the line-source sprinkler system. Soil Science Society American. Journal 44, 886-888.

Harville, D.A. (1977). Maximum likelihood approaches to variance component estimation and to related problems. JASA 72, 320-338. 
Jennrich, R.I., and Schluchter, M.D. (1986). Unbalanced repeated-measures models with structured covariance matrices. Biometrics, 42, 805-820.

Johnson, D.E., Chaudhuri, U.N., and Kanemasu, E.T. (1983). Statistical analysis of line-source sprinkler irrigation experiments and other nonrandomized experiments using multivariate methods. Soil Science Society American Journal, 47, 309-312.

Lindley, D.V.(1972). Bayesian statistics: a review. SIAM.

McLean, R.A., Sanders, W.L., and Stroup, W.W. (1991). A unified approach to mixed linear models. The American Statistician, 45, 54-64.

Marx, D., and Thompson, K. (1987). Practical aspects of agricultural kriging. Bulletin 903, Arkansas Agricultural Experiment Station, Fayetteville.

Schwarz, G. (1978). Estimating the dimension of a model. Annals of Statistics, 6, 461-464.

Searle, S.R., Casella, G., and McCulloch, C.E. (1992). Variance Components, John Wiley \& Sons.

Stroup, W.W. (1989). Use of mixed model procedure to analyze spatially correlated data: an example applied to a line-source sprinkler irrigation experiment. Applications of Mixed Models in Agriculture and Related Disciplines, Southern Cooperative Series Bulletin No. 343, Louisiana Agricultural Experiment Station, Baton Rouge, 104-122.

Swallow, W.H. and Monahan, J.F. (1984). Monte Carlo comparison of ANOVA, MIVQUE, REML, and ML estimators of variance components. Technometrics, $28,47-57$.

Wolfinger, R.D, and O'Connell, M. (1992). Fitting generalized linear mixed models using iteratively reweighted maximum likelihood. In review.

Zimmerman, D.L. and Harville, D.A. (1991). A random field approach to the analysis of field-plot experiments and other spatial experiments. Biometrics, 47, 223-239. 
Table 1: Line-source sprinkler irrigation data

IRRIG

\begin{tabular}{llrrrrrr|rrrrrr} 
B & CULT & 1 & 2 & 3 & 4 & 5 & 6 & 6 & 5 & 4 & 3 & 2 & 1 \\
\hline 1 & Luke & 2.4 & 2.7 & 5.6 & 7.5 & 7.9 & 7.1 & 6.1 & 7.3 & 7.4 & 6.7 & 3.8 & 1.8 \\
1 & Nugaines & 2.2 & 2.5 & 4.3 & 6.3 & 7.9 & 7.1 & 6.2 & 5.3 & 5.3 & 5.2 & 5.4 & 2.9 \\
1 & Bridger & 2.9 & 3.2 & 5.1 & 6.9 & 6.1 & 7.5 & 5.6 & 6.5 & 6.6 & 5.3 & 4.1 & 3.1 \\
2 & Nugaines & 2.4 & 2.5 & 4.0 & 5.8 & 6.1 & 6.2 & 7.0 & 6.4 & 6.7 & 6.4 & 3.7 & 2.2 \\
2 & Bridger & 2.6 & 3.1 & 5.7 & 6.4 & 7.7 & 6.8 & 6.3 & 6.2 & 6.6 & 6.5 & 4.2 & 2.7 \\
2 & Luke & 2.2 & 2.7 & 4.3 & 6.9 & 6.8 & 8.0 & 6.5 & 7.3 & 5.9 & 6.6 & 3.0 & 2.0 \\
3 & Nugaines & 1.8 & 1.9 & 3.7 & 4.9 & 5.4 & 5.1 & 5.7 & 5.0 & 5.6 & 5.1 & 4.2 & 2.5 \\
3 & Luke & 2.1 & 2.3 & 3.7 & 5.8 & 6.3 & 6.3 & 6.5 & 5.7 & 5.8 & 4.5 & 2.7 & 2.3 \\
3 & Bridger & 2.7 & 2.8 & 4.0 & 5.0 & 5.2 & 5.2 & 5.9 & 6.1 & 6.0 & 4.3 & 3.1 & 3.1 \\
\hline
\end{tabular}


Table 2: Results from fitting various mixed models to the line-source sprinkler irrigaton data

\begin{tabular}{|c|c|c|c|c|c|c|c|}
\hline Covariance & Random & REML & REML & REML & ML & ML & ML \\
\hline Structure $(R)$ & Effects $(G)$ & $\mathrm{AIC}$ & $\mathrm{SBC}$ & $-2 \mathrm{LL}$ & $\mathrm{AIC}$ & $\mathrm{SBC}$ & $-2 L L$ \\
\hline I & & -125.4 & -126.6 & 248.8 & -121.1 & -122.4 & 240.1 \\
\hline I & $\mathrm{B} \mathrm{B} \mathrm{B}^{*} \mathrm{CLT}+$ & -116.2 & -120.0 & 226.4 & -110.5 & -114.5 & 215.0 \\
\hline I & B B*IRRIG & -116.2 & -120.0 & 226.4 & -110.5 & -114.5 & 215.0 \\
\hline CS & & -118.9 & -121.4 & 233.8 & -114.6 & -117.3 & 225.2 \\
\hline $\mathrm{AR}(1)$ & & -114.4 & -116.9 & 224.7 & -108.5 & -111.2 & 213.1 \\
\hline $\operatorname{AR}(1)^{*} \mathrm{CULT}$ & & -117.4 & -124.9 & 222.8 & -111.4 & -119.4 & 210.7 \\
\hline $\mathrm{AR}(1)^{*} \mathrm{CULT}+\mathrm{I}$ & & & + & & & $\ddagger$ & \\
\hline TOEP & & -108.7 & -123.7 & 193.5 & -92.2 & -108.3 & 160.5 \\
\hline TOEP(4) & & -113.2 & -118.2 & 218.4 & -106.5 & -111.9 & 205.0 \\
\hline UN & & & $\ddagger$ & & & $\ddagger$ & \\
\hline $\mathrm{UN}(1)$ & & -118.2 & -133.2 & 212.3 & -107.0 & -123.1 & 189.9 \\
\hline CS & $\mathrm{B} \mathrm{B}^{*}$ IRRIG & -116.6 & -121.6 & 225.3 & -110.2 & -115.5 & 212.4 \\
\hline $\operatorname{AR}(1)$ & $\mathrm{B} \mathrm{B}{ }^{*}$ IRRIG & -113.2 & -118.2 & 218.5 & -107.1 & -112.4 & 206.1 \\
\hline TOEP & B B*IRRIG & -105.8 & -123.3 & 183.7 & -98.6 & -117.4 & 169.2 \\
\hline $\operatorname{TOEP}(4)$ & $\mathrm{B} \mathrm{B}^{*}$ IRRIG & -111.4 & -118.9 & 210.8 & -104.1 & -112.2 & 196.6 \\
\hline $\mathrm{UN}(1)$ & B B*IRRIG & & $\ddagger$ & & & $t$ & \\
\hline $\mathrm{SP}(\mathrm{POW})$ & & -107.1 & -109.6 & 210.1 & -99.5 & -102.1 & 194.9 \\
\hline $\mathrm{SP}(\mathrm{POWA})$ & & -112.9 & -116.6 & 219.7 & -106.4 & -110.4 & 206.7 \\
\hline $\mathrm{SP}(\mathrm{SPH})$ & & -111.0 & -113.5 & 217.9 & -105.0 & -107.7 & 206.0 \\
\hline $\mathrm{SP}(\mathrm{GAU})$ & & -115.1 & -117.6 & 226.2 & -108.4 & -111.1 & 212.8 \\
\hline $\mathrm{SP}(\mathrm{LIN})$ & & & $\ddagger$ & & & $\ddagger$ & \\
\hline $\mathrm{SP}(\mathrm{POW})$ & $\mathrm{B}$ & -107.5 & -111.2 & 209.0 & -99.9 & -104.0 & 193.9 \\
\hline $\mathrm{SP}(\mathrm{POWA})$ & B & -111.0 & -116.0 & 214.0 & -104.1 & -109.5 & 200.3 \\
\hline
\end{tabular}

$\dagger$ Variance component for $\mathrm{B}^{*} \mathrm{CULT}$ is zero and removed from remaining models + Did not converge 